\title{
Supramolecular gel: From structure to function
}

With the development of molecular science, which focuses largely on the synthesis and functions of molecules linked together by covalent bonds, interest in supramolecular assemblies has increased. Supramolecular assemblies comprise ordered molecular systems that are held together by the cooperative or synergetic effects of non-covalent interactions such as hydrogen bonding, $\pi-\pi$ stacking, electrostatic interactions, coordination, and hydrophobic interactions. Great effort has been devoted to designing and fabricating various supramolecular assemblies to produce functional soft materials that can be used in optoelectronic devices, smart responsive materials, high-performance catalytic systems, biomimetic and/or biocompatible materials and drug delivery systems. To date, many supramolecular functional systems have been constructed using "bottom-up" strategies, such as interfacial organization, surfactant-assisted assembly, molecular recognition, evaporation, electro-spinning and gelation techniques.

Among the various assembly methods and molecular organized systems, gelation and gel systems have been intensely studied in recent years. Although polymeric gels have been investigated for a long time, the new low-molecular weight gels (organogels and hydrogels) have drawn renewed interest because of their facile fabrication and variety in design and functionalization. A variety of different gels and structurally organized nanomaterials can be obtained either through a simple heating and cooling process, or other stimulus of the gelator solutions. Because of the weak non-covalent interactions, the gels reversibly change between individual molecules and assemblies and are involved in assembly/disassembly processes. These fascinating characteristics of supramolecular gels allow them to be used in bionics, drug delivery systems, and smart materials. Furthermore, various well-defined nanostructures with controllable morphology, composition and even physicochemical properties can be easily synthesized in large quantities using supramolecular gelation processes. Thus, supramolecular gels are currently a hot topic and supramolecular science which involves collaborations between chemists, physicists, material scientists and engineers is evolving into an attractive interdisciplinary field.

To promote the research and exchange ideas in the material science field in China, we organized a workshop at Shaanxi Normal University, Xi'an (8-10 November, 2011) under the support of the Chinese Chemical Society. In this forum, more than 50 scientists from Chinese universities and institutes discussed the history, current status, and the future trends in supramolecular gels and relevant functional materials. The forum is a great success and addresses many important issues.

This special topic reports the results presented at the workshop and represents the recent progress made in the field of material sciences in China. We believe that these results will be of interest to the scientific and technological communities.

LIU MingHua
Center for Molecular Science, Institute of Chemistry, Chinese Academy of Sciences, Beijing 100190, China

FANG Yu

Key Laboratory of Applied Surface and Colloid Chemistry, Shaanxi Normal University, Xi'an 710062, China

Open Access This article is distributed under the terms of the Creative Commons Attribution License which permits any use, distribution, and reproduction in any medium, provided the original author(s) and source are credited. 